\title{
Electron microscopy in the cytological examination of metastatic pleural effusions
}

\author{
M. LEGRAND and R. PARIENTE \\ Hôpital Antoine Béclère, 157 rue de la Porte de Trivaux, F92140, Clamart, France
}

\begin{abstract}
Legrand, M. and Pariente, R. (1970). Thorax, 31, 443-449. Electron microscopy in the cytological examination of metastatic pleural effusions. In a previous publication the ultrastructure of pleural effusions in cases of pleural mesothelioma was reported. The same method has now been applied to a study of effusions produced by pleural metastases. The findings are considered sufficiently conclusive to justify the use of electron microscopic cytology in determining the nature and sometimes the origin of such effusions.
\end{abstract}

Sixty-three pleural fluids from patients with metastatic pleural involvement were examined by electron microscopy using the techniques previously described by Legrand and Pariente (1974).

\section{RESULTS}

Numerous tumour cell types were observed but all had common characteristics irrespective of their degree of differentiation. The tumour cells occurred both singly and in clusters and varied from a few to many. At low magnification the groups of cells took on the form of rosettes (Fig. 1) or appeared as tissue fragments (Fig. 2). Binucleated cells (Fig. 3), sometimes containing vacuoles or cellular enclosures (Fig. 4), were often seen. Junctional complexes and the character of the cell surface gave evidence of cell differentiation. Some cells showed numerous upright short rectangular microvilli, 0.8 to $1 \mu$ long, with a glandular structure. Dense granules, varying in size from 100 to $300 \mathrm{~m} \mu$, were sometimes seen at the periphery of the cells near groups of microvilli and resembled a secretory zone (Fig. 5). The cytoplasm often contained some clear homogeneous vacuoles, the contents of which were probably extracted during the fixation (Fig. 4).

The mitochondria were frequently altered and the endoplasmic reticulum was often smooth, fragmented, and swollen. Free ribosomes were abundant. The nuclei were irregular, containing one or more nucleoli and some nuclear bodies. Intranuclear cytoplasmic expansions were numerous, occasionally giving a fragmented appearance to the nuclei.

The cells in the pleural effusion of patients with carcinoma of the breast were single or in clusters, sometimes forming a corona around a large central space, as in Fig. 1, and were joined together by numerous desmosomes. Short, straight microvilli occurred at the cell periphery, some isolated cells having microvilli along the entire periphery and others only at one pole. Here the cytoplasm contained dense secretory granules (Fig. 3). In the cell clusters intercellular spaces lined with microvilli and limited by a junctional complex were also found as in a canalicular structure (Fig. 1). The cytoplasm contained numerous mitochondria, often altered, fragments of ergastoplasm, and cisterns of Golgi. Lipid inclusions, often extracted in part by the fixation, were especially characteristic. In some areas small intracellular cavities were seen, bordered by microvilli and containing a dense product. In the adjacent cytoplasm secretory granules were observed (Fig. 5). The cytoplasm also contained glycogen accumulations, but no fibrillary complexes were evident.

Pleural effusions associated with gastrointestinal tumours showed mainly groups of cells representing fragments of cellular tissue, and single cells were uncommon (Fig. 2). The cytoplasm contained many lipid droplets as well as dense inclusions at the periphery of the cell. Mitochondria were small, numerous, and clustered around the nucleus. The ergastoplasm formed long cisterns spread throughout the cytoplasm. The particularly fragmented nucleus was sometimes double, containing a clear chromatin and some chromatid bodies. Dense secretory granules were distributed under the cell membrane which was lined with short, straight, clubbed microvilli.

Pleural effusions due to metastases from a pancreatic tumour were characterized by cells with an abundance of rough endoplasmic reticula arranged 


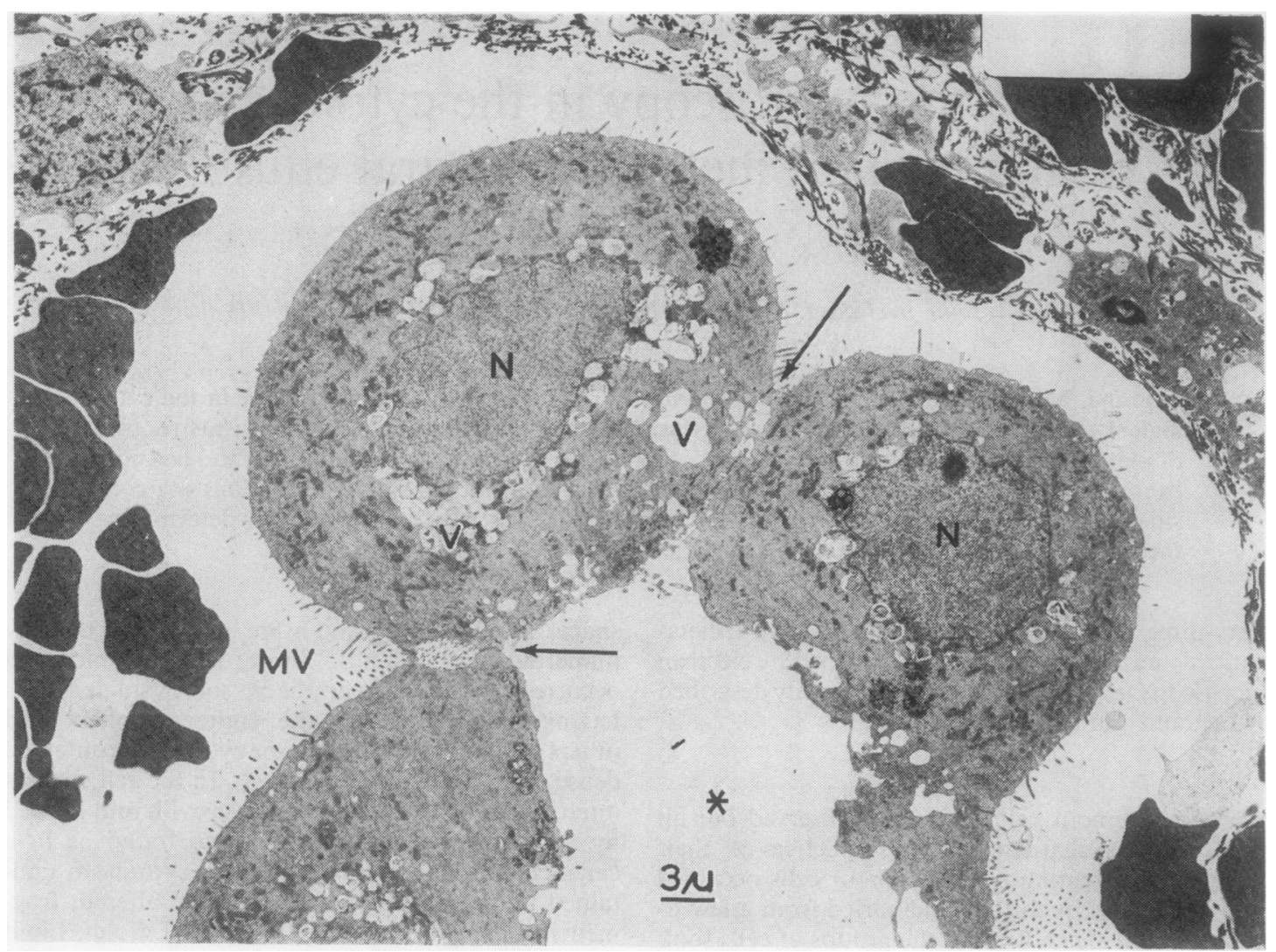

FIG. 1. A group of metastatic cells from a breast cancer. There is a clear space between the tumour cells and the usual cells found in a pleural effusion. The tumour cells have a border of small, short microvilli $(M V)$. The nucleus $(N)$ is clear and bordered by lipid vacuoles $(V)$. The cells are joined together by desmosomes (arrow). An intercellular cavity is visible $(*)$. $(\times 2430)$.

in parallel or circular lines (Fig. 4). There were few mitochondria but numerous vacuoles of varying size. No secretory granules were observed. The cells were frequently enclosed without junctional complexes between them. The nuclei were irregular and contained dense chromatin at the periphery and numerous nuclear bodies. Some microvilli were visible at the periphery and in the intercellular spaces.

The less differentiated glandular tumours that metastasized to the pleura produced an effusion with single or groups of cells which were of ten enclosed in each other (Fig. 6). They contained secretory granules, the presence of which with a size of less than $200 \mathrm{~m} \mu$ suggested an endocrine origin.

The tumours corresponding to the papillary type observed on light microscopy showed cellular clusters that could be confused with mesothelial cells. They had in fact abundant, long, sinuous microvilli of the mesothelial type and a nucleus with fine chromatin and a large spiral nucleolus. The cytoplasm was, however, different, having no peri- $\odot$ nuclear fibrils, very abundant fine and regular $N$ ergastoplasm, a very voluminous Golgi apparatus, N and numerous free ribosomes. These aspects were strongly suggestive of biosynthetic activity corresponding to glandular tumours of ovarian or thyroid origin.

Some pleural fluids contained malignant cells of undetermined origin, although certain of their characteristics suggested a metastatic origin. Changes in the cytoplasmic bodies and an absence of surface differentiation did not correspond with any known 


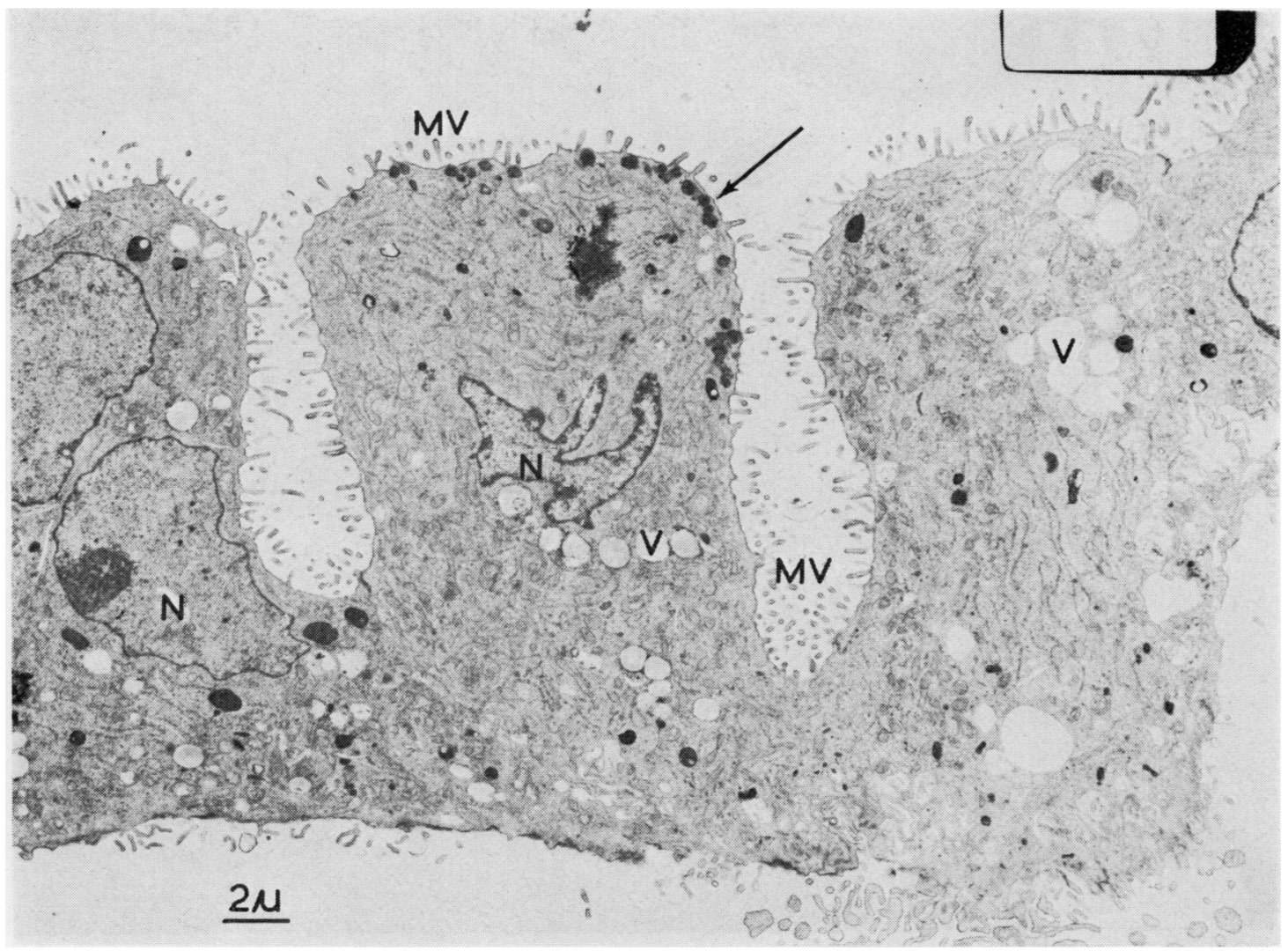

FIG. 2. A group of metastatic cells from a colonic carcinoma. These cells are fragments of cellular tissue. Their cytoplasm contains numerous clear lipid inclusions $(V)$ and some secretory granules at the apical pole (arrow). The mitochondria are numerous and the ergastoplasm forms long unorganized cisterns. The nuclei are indented or divided $(N)$. At the apical pole the cell membrane is lined with numerous short microvilli $(M V)$. On the cell in the centre of the field, the extremity forms a true secretory zone (arrow) $(\times 4150)$.

primary. Their metastatic nature could, however, be assumed when there were enclosed cells and a rough ergastoplasm indicating glandular activity. In these cases the absence of differentiation on subsequent examination confirmed their metastatic origin.

\section{DISCUSSION}

The electron microscopic examination of pleural fluids has not been carried out systematically up to now. In an earlier report we presented some pre- liminary results (Legrand et al., 1972) and then applied the method to the study of the pleural fluids of mesotheliomas (Legrand and Pariente, 1974). In practice, the examination of pleural fluid is simple to perform and repeat. Samples can be obtained readily by pleural aspiration. Centrifugation does not damage the cells and a higher cellular concentration is obtained. The electron microscope allows more accurate characterization of the cell than is possible by light microscopy. Errors can, however, arise. False conclusions may result from confusing macro- 


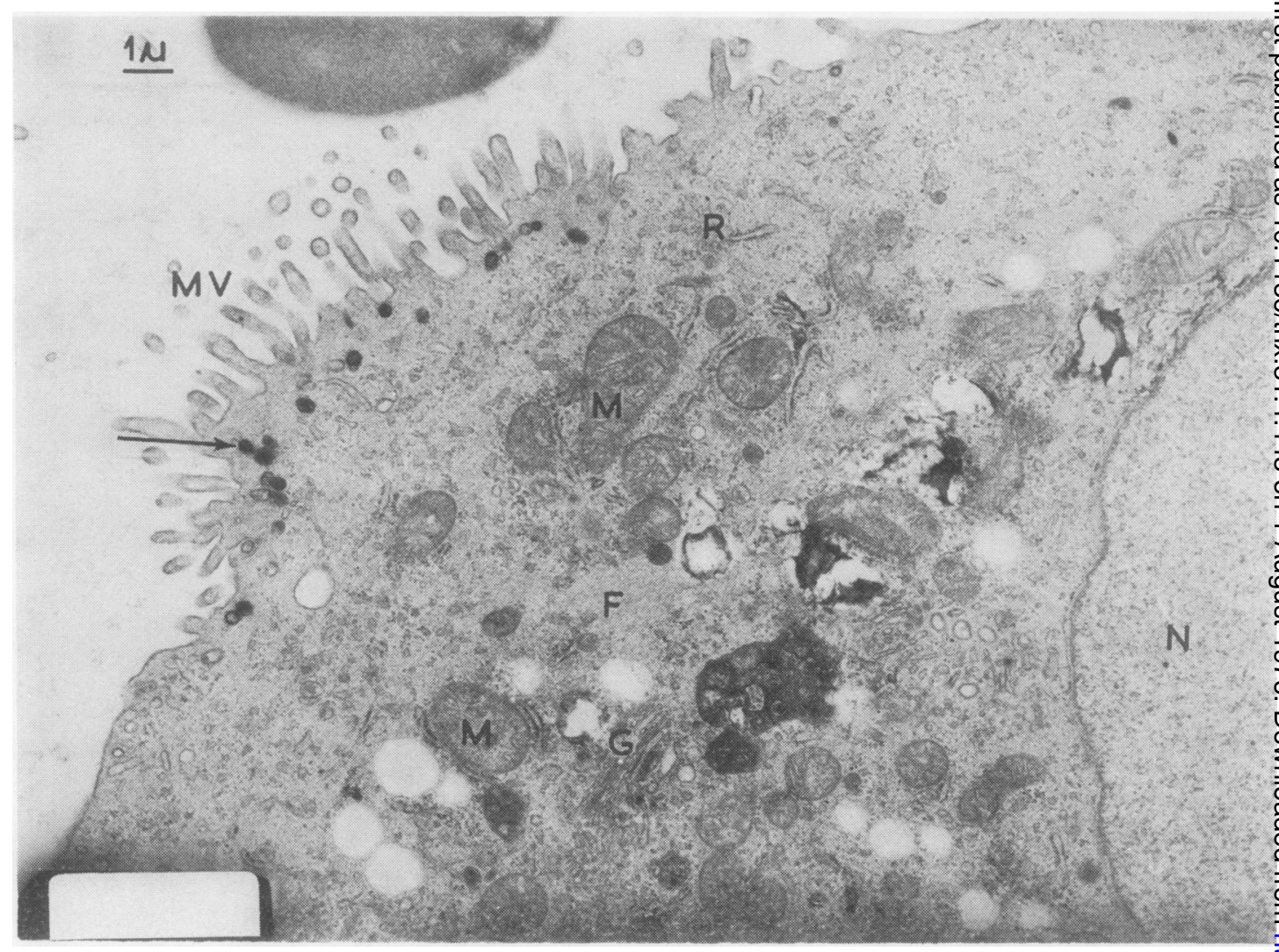

FIG. 3. The secretory zone of a metastatic breast cancer cell. The cell membrane is lined with a cluster of microvilli $(M V \overline{9}$ under which some electron-dense secretory granules are visible (arrow). In the cytoplasm the reticulum forms short fragments $(R)$. The mitochondria are normal $(M)$. The Golgi apparatus is well developed $(G)$. The cytoplasm contains some fine fibrils $(F) \underline{\underline{Q}}$ This appearance of the secretory pole is common in single cells from a metastasis of a breast carcinoma $(\times 13800)$.

phages with mesothelial cells, particularly in chronic effusions. Macrophages are large and have an irregular nucleus with cytoplasmic inclusions which can be misinterpreted as lipid inclusions. However, their long, confluent, irregular pseudopodia are different from the usual malignant cell microvilli and there is no change in the cytoplasmic bodies nor is there any large intracellular cavity: moreover, phagocytosis with lysis of the absorbed cell is unlikely to be confused with the enclosure of one cell by another (Fig. 6).

The mesothelial cells of a chronic effusion are sometimes difficult to identify with certainty due to changes in the mitochondria and the reticulum. They may contain lipid inclusions similar to those tumour cells but have neither secretory granules no nuclear irregularities. They do not form groups of tissue cells nor show cellular enclosures or intra ${ }^{N}$ cellular cavities. Their appearance is unchanged ort repeated examination. By contrast, secondary cance cells show pyknotic changes which often make if difficult to recognize the cytoplasmic structures Spriggs and Meek (1961) have drawn attention to the neoplastic characteristics of microvilli clustered ato the pole of a cell and differing from pseudopodiao These microvilli are identifiable even at low magni令 fication. Ebner and Schneider (1956) observed thष्ठ same microvilli in ovarian tumour cells present ig 


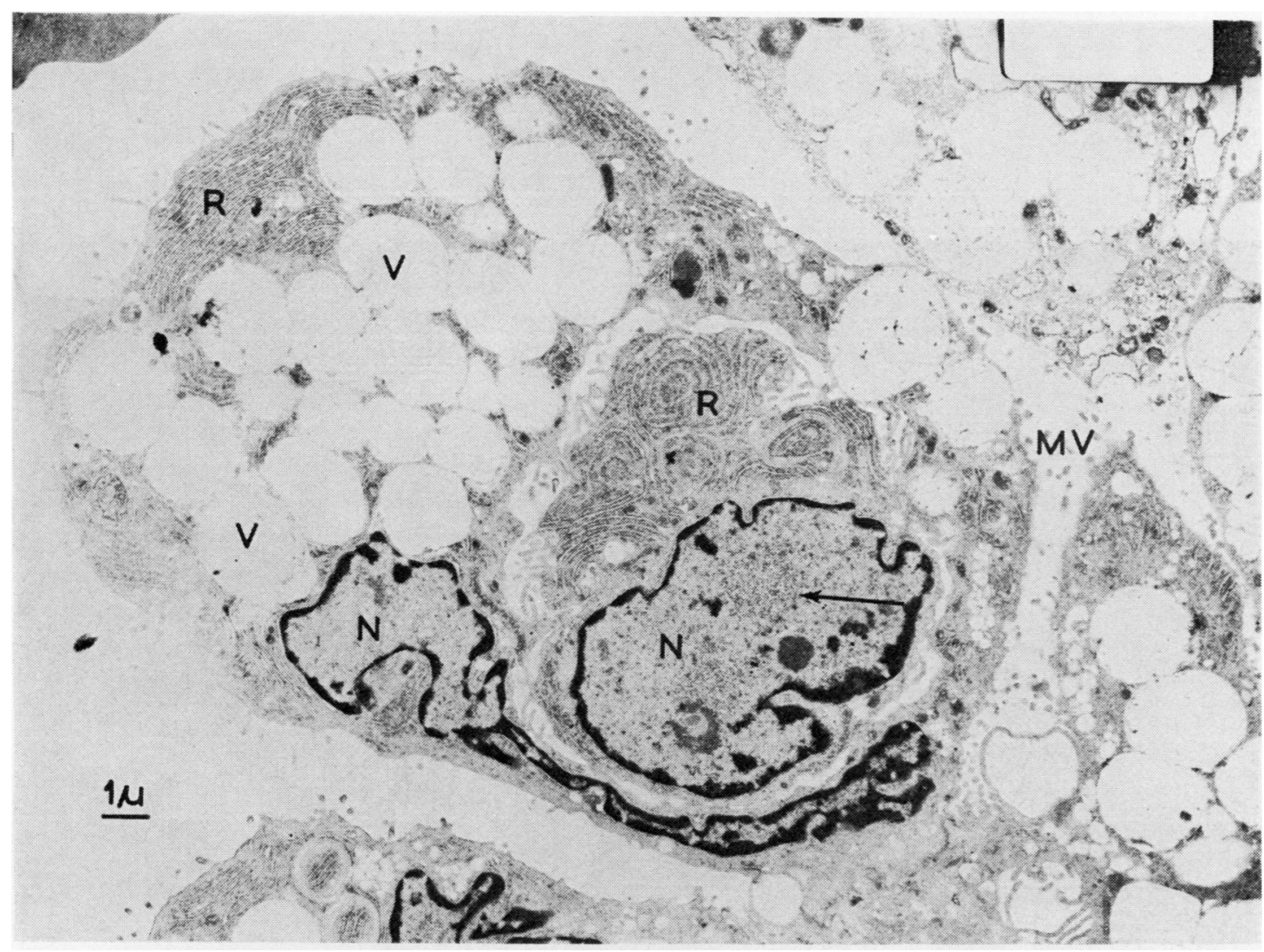

FIG. 4. A group of metastatic cells from a pancreatic carcinoma. Note the enclosure of the central cell in another cell. There are no desmosomes in the intercellular space, which contains some microvilli $(M V)$. The nuclei $(N)$ of the outer cells are irregular, surrounded by dense and irregular chromatin. The nucleus of the central cell is notched by the cytoplasm. It contains fragments of nucleolus and some abnormal nuclear bodies (arrow). The cytoplasm contains numerous clear vacuoles of varying size $(V)$. These probably contained lipid which was extracted during fixation with osmium. The rough reticulum $(R)$ is very abundant, clustered in parallel or circular cisterns $(\times 6900)$.

ascitic fluid. Their short, straight appearance is never seen in non-malignant pleural cells and constitutes a criterion for recognizing metastatic cancer cells, especially those of glandular origin. Although metastatic cells are unlikely to be confused with fibroblast-like mesothelial cells (Legrand and Pariente, 1974), errors are possible between the socalled papillary tumours and mesotheliomas. The microvilli are similar but metastatic cells never have either a concentric structure or a fibrillary perinuclear condensation. The cells of papillary tumours have more ergastoplasm and free ribosomes and never show fibroblastic differentiation. Among the characteristics of secondary cancer, two are sufficiently specific to be of great importance in diagnosisshort, straight microvilli and the enclosure of cells. The structure of enclosed cells is usually well preserved and the membranes are often joined together by junctional complexes. These appearances are probably due to artifacts in the preparation of the sections. In doubtful cases examination of the pleural fluid should be repeated. 
FIG. 5. Intracellular cavity in a metastatic breast cancer cell. This cavity is bordered by numerous microvilli. At the centro of the cavity there is a dense and homogeneous secretory product $\left(^{*}\right)$. At the periphery the cytoplasm contains some dense secretory granules bordered by a membrane (arrow), some unaltered mitochondria $(M)$, and free ribosomes. No ergastoplasm is seen. The cytoplasm contains many fine fibrils $(F)(\times 17250)$. 


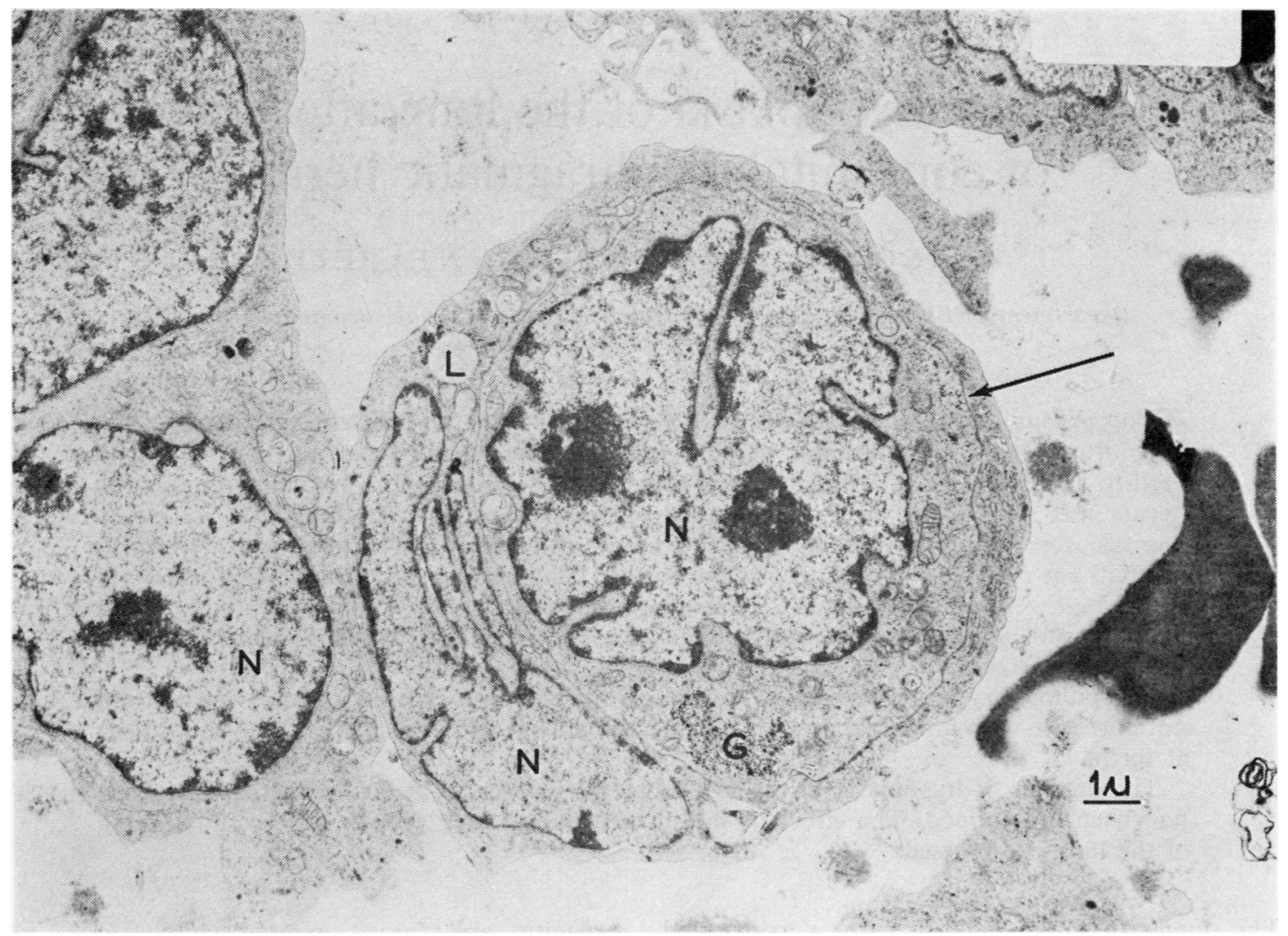

FIG. 6. Slightly differentiated metastatic tumour cells. The nuclei are irregular and contain cytoplasmic extensions $(N)$. The chromatin is dense and irregular at the periphery. Several large nucleoli are visible. The cellular membranes are not lined with microvilli. The intercellular spaces (arrow) are rectilinear with some occasional small junctions. The cytoplasm contains little ergastoplasm, some normal and altered mitochondria, an accumulation of glycogen $(G)$, and lipid $(L)$ inclusions. The central cell with two nucleoli is enclosed but does not appear pyknotic. The enclosure of one cell in another confirms the diagnosis of metastases despite the absence of differentiation $(\times 6900)$.

\section{REFERENCES}

Ebner, H. and Schneider, W. (1956). Zur Zytologie eines menschlichen Aszitestumor. Zentralblatt für Gynäkologie, 78(2), 1486.

Legrand, M., Andre-Bougaran, J., and Pariente, R. (1972). Examen cytologique des liquides pleureux au microscope électronique. Revue de Cytologie Clinique, $5,151$.

and Pariente, R. (1974). Ultrastructural study of pleural fluid in mesothelioma. Thorax, 29, 164.
Spriggs, A. I. and Meek, G. A. (1961). Surface specialisations of free tumour cells in effusions. Journal of Pathology and Bacteriology, 82, 151.

Requests for reprints to: Dr. M. Legrand, Centre de Pneumologie, Hôpital Beaujon, F-92110, Clichy, France. 